\title{
Pregnancy in a Woman with a Congenital Type of Nemaline Myopathy
}

\author{
Binder Tomášs ${ }^{1, *}$, Gerych Pavel ${ }^{1}$, Škola Josef ${ }^{2}$, Beneš Jan², Alwail Abdulhamid ${ }^{3}$, \\ Stádníková Milena ${ }^{3}$, Hitka Patrik ${ }^{4}$, Laštůvková Jana ${ }^{5}$ \\ ${ }^{1}$ Department of Gynecology and Obstetrics, Jan Evangelista Purkyně University and the Masaryk Hospital, Ústí nad Labem, Czech Republic \\ ${ }^{2}$ Department of Anesthesiology, Perioperative and Intensive Care of the Jan Evangelista Purkyně University and the Masaryk Hospital, Ústí \\ nad Labem, Czech Republic \\ ${ }^{3}$ NEMOS PLUS s.r.o., Ostrov Hospital, Follow-up Intensive Care, Ostrov, Czech Republic \\ ${ }^{4}$ Neonatology Department, Jan Evangelista Purkyně University and the Masaryk Hospital, Ústí nad Labem, Czech Republic \\ ${ }^{5}$ Department of Medical Genetics of the Masaryk Hospital, Ústí nad Labem, Czech Republic
}

\section{Email address:}

tomas.binder@kzcr.eu (B. Tomáš)

${ }^{*}$ Corresponding author

\section{To cite this article:}

Binder Tomáš, Gerych Pavel, Škola Josef, Beneš Jan, Alwail Abdulhamid, Stádníková Milena, Hitka Patrik, Laštůvková Jana. Pregnancy in a Woman with a Congenital Type of Nemaline Myopathy. Journal of Gynecology and Obstetrics. Vol. 9, No. 5, 2021, pp. 167-171. doi: $10.11648 /$ j.jgo.20210905.16

Received: August 25, 2021; Accepted: September 15, 2021; Published: October 12, 2021

\begin{abstract}
Introduction: Congenital nemaline myopathy is a genetically heterogeneous disease caused by gene defects in a number of genes: nebulin (2q21-22) alpha-tropomysin (1q21-23), alpha-actin (1q42) and others. Nemaline myopathy can be inherited as an autosomal recessive or dominant trait. Case report: We present a case of the first pregnancy in a woman with a congenital type of nemaline myopathy, with a severe pulmonary involvement, in the Czech Republic. Course of pregnancy: The woman got spontaneously pregnant less than two months after a respiratory failure, when she had to be tracheostomized, remained dependent on portable ventilator support. The patient strictly refused artificial abortion recommended to her. Until the $22^{\text {nd }}$ gestational week she was stabilized in terms of ventilation and received health care at home. Subsequently, she was repeatedly admitted to the hospital for lung infection to ICU of the Department of Intensive Care. The fetus was developing proportionately, without signs of muscle hypotonia, but after the $23^{\text {rd }}$ g.w. was diagnosed with slightly progressive polyhydramnios. Childbirth: It was decided to schedule termination of the pregnancy by a caesarean section for the $30^{\text {th }}$ g.w., during the interval of the patient's relatively satisfactory clinical respiratory and mental stabilization. A boy was born $(1120 \mathrm{~g})$ and was transferred to the NICU. The newborn required artificial pulmonary ventilation, from the $3^{\text {rd }}$ day he was extubated, with intermittent use of nasal CPAP until the $9^{\text {th }}$ day. Results: The baby prospered, tolerated food and showed no signs of muscle hypotonia. A postoperative course of the mother went without any complications. On the $8^{\text {th }}$ postoperative day she was discharged from the hospital, ventilator-dependent and with tracheostomy in place. Conclusion: Pregnancy in women with a congenital type of nemaline myopathy with a severe lung involvement is a challenge for physicians. Based on the course of pregnancy it is necessary to decide when its termination will be safe for both the mother and the fetus. The care of the patient requires a multidisciplinary approach.
\end{abstract}

Keywords: Nemaline Myopathy, Pregnancy, Respiratory Failure

\section{Introduction}

Congenital nemaline myopathy is a genetically heterogeneous disease caused by gene defects in a number of genes: nebulin (2q21-22) alpha-tropomysin (1q21-23), alpha- actin (1q42) and others [10]. Nemaline myopathy can be inherited as an autosomal recessive or dominant trait. Nemaline myopathy is a disorder that primarily affects skeletal muscles, which are muscles that the body uses for movement. People with nemaline myopathy have muscle 
weakness (myopathy) throughout the body, but it is typically most severe in the muscles of the face; neck; trunk; and other muscles close to the center of the body (proximal muscles), such as those of the upper arms and legs. This weakness can worsen over time. Affected individuals may have feeding and swallowing difficulties, foot deformities, abnormal curvature of the spine (scoliosis), and joint deformities (contractures). Most people with nemaline myopathy are able to walk, although some affected children may begin walking later than usual. As the condition progresses, some people may require wheelchair assistance. In severe cases, the muscles used for breathing are affected and life-threatening breathing difficulties can occur. Nemaline myopathy is divided into six types. In order of decreasing severity, the types are severe congenital, Amish, intermediate congenital, typical congenital, childhood-onset, and adult-onset. The types are distinguished by the age when symptoms first appear and the severity of symptoms; however, there is overlap among the various types. The severe congenital type is the most lifethreatening. Most individuals with this type do not survive past early childhood due to respiratory failure. The most common type of nemaline myopathy is the typical congenital type, which is characterized by muscle weakness and feeding problems beginning in infancy. Most of these individuals do not have severe breathing problems and can walk unassisted. Pregnancy in these women is very rare, requires the care of a multidisciplinary team. The course is often complicated with polyhydramnios. $30-50 \%$ of pregnancies end in preterm birth. $[2-6,9]$

\section{Case Report}

A 23-year old female patient E. H, I/0, height $143 \mathrm{~cm} /$ body weight $35 \mathrm{~kg}$.

\subsection{History}

\section{a) Personal History}

The patient suffers from a congenital type of nemaline myopathy. She has had problems since childhood, and was repeatedly treated for respiratory infection. She has a cachectic physiognomy, with a significant stigmatization of the face: elongated face with low-set, sticking out ears, high arched palate, sinistroconvex scoliosis of the spine with hyperlordosis. The lower extremities show hypotrophy of muscles of the right lower leg, cavus foot with internal rotation laterally, shortening of the Achilles tendon bilaterally. She has a markedly limited mobility, but is able to walk without assistance, with stabilization boots and a crutch. Myopathy was confirmed by biopsy of the lower leg muscles in 2006, but confirmation of the diagnosis by DNA is absent. Echocardiography demonstrated moderate mitral regurgitation with anomaly of papillary muscles and thickening of the anterior leaflet of the mitral valve, moderate tricuspid regurgitation. She suffers from chronic hypercapnic respiratory insufficiency and bronchial asthma. Since 2014 she has intermittently required NIV BiPAP before bedtime. b) Family History

3 healthy biological siblings, father - asthmatic, grandfather - lung cancer, mother - healthy.

c) Social History

Single, disability pensioner, living with her mother.

d) Allergy

To cow milk and eggs.

e) Addiction

The patient smoked until 2014, including experimenting with marijuana and pervitin.

f) Gynecological History

Menarche at the age of 11 years, regular menstrual cycle, the last menstrual period (LMP): 19 September 2019.

\subsection{The Pre-pregnancy Status}

On July $12^{\text {th }} 2019$, the patient was admitted to the Department of Pulmonary Diseases, Masaryk Hospital, Ústí $\mathrm{n}$. Labem, for lateral pneumonia and respiratory insufficiency. She received antibiotic therapy with cefotaxime and clarithromycin. Her condition was gradually deteriorating, with hypotension, tachycardia and progressing respiratory insufficiency. On the second day she was transferred to ICU of the Department of Intensive Care, intubated and artificial pulmonary ventilation and bronchoscopic sputum suction were introduced. Echocardiography confirmed severe pulmonary hypertension with cor pulmonale. The patient's condition required application of tracheostomy, introduction of the central venous catheter, nasogastric tube and an indwelling urinary catheter. After 10 days of intensive care, the patient's condition gradually stabilized and she could be disconnected from the artificial pulmonary ventilation, although she still required ventilation support by a portable ventilator. Due to uncertain diagnosis and increased risk of nosocomial infection, she was transferred to the Department of Follow-up Intensive Care for rehabilitation and weaning.

Upon admission the patient was supported by controlled ventilation, $\mathrm{SpO}_{2}$ 92\% with $\mathrm{FiO}_{2}$ 0.4; abundant amount of whitish sputum was removed by suction; further circulatory support with noradrenaline was necessary; blood pressure $115 / 50$, regular heart rate; ECG finding without ischemic changes; nasogastric tube without discharge; indwelling urinary catheter draining clear urine; positive MRSA nasal swab; high values of laboratory tests: TSH 10.9, hypalbuminemia 21.6, CRP 126; predominating marked muscle weakness; absence of pressure ulcers on the body. Elevated inflammatory parameters required continuation of ATB therapy (Sefotak, Citalopram, Mirtazapin). Breathing problems deteriorated by the patient's attacks of panic anxiety were addressed by administration of Berodual, Abtrovent and expectorants, while the panic anxiety itself was treated with the use of antidepressants. Hypothyroidism was compensated by levothyroxine (Letrox) substitution therapy. LMWHs (Clexane $0.2 \mathrm{ml} / \mathrm{sc} / 24 \mathrm{~h}$ ) were applied throughout the whole period of the patient's hospital stay to prevent thromboembolic disease. The follow-up CT scan of the lungs and mediastinum confirmed pneumonia of the lower lobe of the left lung, showing varicose bronchiectasis 
with multiple air-filled lesions, a slight regression of inflammatory changes, a mild fluidothorax and a $16 \times 12 \mathrm{~mm}$ subpleural bulla at the right lung apex. Laboratory tests showed gradual reduction of inflammatory markers. The patient remained ventilator dependent $\left(\mathrm{FiO}_{2}\right.$ 0.3). Her condition was getting stabilized, without episodes of sudden breathlessness. She was cooperating; during the following period she underwent an intensive rehabilitation, ergotherapy and received nutritional support. This was followed by the patient's gradual verticalization, including gait training. At the end of the hospital stay she was self-sufficient and continent. The patient was discharged from the hospital care on the $17^{\text {th }}$ of September 2019. Within one month after the discharge she got spontaneously pregnant.

\subsection{Trimester Course of Pregnancy}

On December $10^{\text {th }} 2019$, the patient and her mother visited the High Risk Pregnancy Center of the Department of Gynecology and Obstetrics at the Masaryk Hospital. She was diagnosed with pregnancy, with gestational age of $10+5$ weeks. The patient was portable ventilator-dependent, tracheostomized. Both the patient (and her mother) were informed that with the patient's condition, pregnancy might be life-threatening, carrying the baby to term could not be expected and, on the contrary, that there was a high uncertainty as for a viable child outcome. Artificial abortion was suggested due to the patient's vital indication, which was strictly refused by the patient. First trimester combined screening and genetic examination were recommended. At that time, the patient was without any significant subjective complaints and was relatively stabilized. The result of the first trimester screening was negative.

\subsection{Genetic Examination}

Analysis of the genes associated with the neuromuscular disease proved two likely pathogenic sequence variants, probably in trans.

1. Gene NEB (LRG_202t1: c.2211+5G Ap? in heterozygous configuration.

2. Gene NEB (LRG_202t1): c.13059+5G Ap? in heterozygous configuration.

The proband showed a normal 46, XX female karyotype; aCGH test revealed no significant deletion and no duplication of chromosomes. The proband's partner is healthy, without consanguinity. Therefore the risk of inheritance of congenital nemaline myopathy for the fetus was probably genetically favorable; it could be further specified by a targeted molecular genetic analysis of the amniotic fluid, which was refused by the proband.

\subsection{Trimester Course of Pregnancy}

Until $22^{\text {nd }}$ g.w., the patient was stabilized and received health care at home. The course of pregnancy was uneventful. The fetus was doing well. The 20 g.w. ultrasound screening showed no apparent gross pathology. Fetal echocardiography demonstrated no structural changes. Cachexia in the patient was persisting (body weight $35 \mathrm{~kg}$ ), together with a general muscle weakness. She was quite self-sufficient, with a limited mobility. A regularly visiting field nurse was in charge of exchanging tracheostomy cannulas and removal of mucus from the respiratory tract. At the $22+3$ gestational age, the patient's mother contacted by phone the High Risk Pregnancy Center as she was no longer able to handle the situation at home. A stat test was recommended. Despite the ventilation support, the patient had breathing difficulties during any activity, gynecological examination could not be performed as the patient was unable to lie supine because she could not breathe and had panic attacks. On the $3^{\text {rd }}$ of March 2020, the patient was admitted to the High Risk Pregnancy ward and after consultation with the intensivist transferred to ICU the next day. There she received antibiotic therapy with Cefuroxime and her vital functions were monitored. The patient continued to have persistent positive MRSA blood cultures. After adjustment of ventilation support parameters, her condition stabilized relatively quickly. In spite of this, fetal lung maturation was accelerated by corticosteroids (Diprophos) in view of uncertain pregnancy prognosis. Ultrasonography showed a well developing fetus, with a normal motion; the amount of amniotic fluid was adequate. Due to a high risk of nosocomial infection, on March 10th the patient was transferred to health care at home. After one week on the $18^{\text {th }}$ of March 2020, the patient was readmitted to ICU of the Department of Intensive Care, for exacerbation of respiratory insufficiency. Initially the condition was managed by adjustment of the ventilation support protocol, without necessity of ATB therapy based on negative sputum culture results. On March 31st, the patient's condition deteriorated, with elevation of inflammatory markers. Her mental status altered again, she ceased to cooperate and refused attachment of the ventilator. Subsequently, nosocomial pneumonia was confirmed and the sputum culture proved the presence of Staphylococcus aureus (MRSA), Hemophilus influenzae, Staphylococcus pneumoniae and Moraxella catarrhalis. A combined therapy with piperacillin/tazobactam antibiotics was introduced and subsequently adjusted in view of sensitivity to ceftaroline. Respiratory and ventilation parameters were gradually adjusted, inflammatory markers decreased, but anemia significantly progressed ( $\mathrm{Hb} 8.2 \mathrm{~g} \%$ ). The patient's mental status became stabilized. Regular gynecologic follow-up demonstrated no progression of the finding in the uterine cervix, but repeated ultrasonography showed a progressing finding of polyhydramnios (AFI 28). The fetus was without signs of hydrops, muscle hypotonia or muscle contractures but there were signs of interuterine stagnation of fetal growth (FGR). A booster Diprophos dose was applied to accelerate fetal lung maturation. Both virological and serological tests of the mother were negative. The cause of polyhydramnios remained unclear, but it develops in connection with myopathy $[1,7]$.

Based on evaluation of the course of the pregnancy and consideration of the risks of repeated nosocomial lung infection, it was decided to schedule its termination for 30 
g.w., taking advantage of both the stabilized respiratory status $\left(\mathrm{SpO}_{2} 96 \%\right)$ and mental status of the patient. Results of control blood tests made on the $13^{\text {th }}$ of April 2019, showed again the elevated level of CRP (31). Therefore and furthermore, at the height of Covid-19 pandemic, the term of cesarean section was hastened. Prior to operation, 2 units of red blood cell mass were administered to correct marked anemia.

\subsection{Childbirth}

On the $14^{\text {th }}$ of April 2019, caesarean section was performed under general anesthesia, using the Küstner's technique. An immature male infant was delivered. After milking (blood from umbilical cord using fingers pushed into the body of the immature), the lively crying newborn was transferred to the care of neonatologists. The surgery and the postoperative course were without complications; on the $3^{\text {rd }}$ day after surgery the patient was transferred in a stabilized condition from ICU of the Department of Intensive Care to the Postpartum Unit. Lactation was discontinued by medicaments. On the $8^{\text {th }}$ postoperative day, the patient was discharged home in a stabilized condition, with a portable ventilator support, and with treacheostomy in place.

Throughout the period of hospital stay and at home she received LMWH to prevent thromboembolic complications, and hypothyroidism was compensated by Letrox substitution therapy $(75 \mathrm{mg} /$ day). She was recommended to continue intensive rehabilitation to be self sufficient and able to take care of the child in the future.

\subsection{Newborn}

A boy was delivered by caesarean section at the 29 g.w., with the birth weight of $1120 \mathrm{~g}$. After the first cry at birth, it was necessary to apply artificial pulmonary ventilation with the use of Neopuff for hypoventilation, followed by intubation and administration of surfactant. He was then transferred to NICU with artificial pulmonary ventilation. Vascular access was created, followed by priming and drips, and antibiotics administered due to initial leukopenia. On the $3^{\text {rd }}$ day, the patient was extubated, with ventilation supported by nCPAP distension therapy. Antibiotic therapy was discontinued, adnate infection was not detected. Drips were terminated after 7 days, with full enteral feeding from then on. Distension therapy could be terminated on the $9^{\text {th }}$ day. On the $15^{\text {th }}$ day, increased oxygen consumption associated with the developing bronchopulmonary dysplasia necessitated reintroduction of nCPAP distension support. Anemia was corrected by red blood cell transfusion. From the $26^{\text {th }}$ day, oxygenotherapy was only minimal, ultrasonography demonstrated a finding adequate to the gestational age, without signs of intraventricular hemorrhage or ischemia. In view of the proved autosomal recessive type of inheritance of nemaline myopathy in the mother, it is obvious that the infant is minimally heterozygous in terms of this myopathy. Therefore the boy will undergo neurological examination.
DNA testing is also recommended, but the mother refuses to consent to this examination. Currently the newborn is doing well, has no signs of muscle hypotonia, after two months of life he reached the body weight of $2650 \mathrm{~g}$.

\section{Conclusion}

Pregnancy in women with a congenital type of nemaline myopathy is extremely rare. In women with a concomitant involvement of lungs, pregnancy is life-threatening and a viable pregnancy outcome is highly uncertain. Logically, this condition is indicated for artificial abortion during the first trimester of pregnancy [1]. In our case, the patient strictly refused such recommendation. It is hard to speculate whether her choice was driven by a strong maternal instinct or whether she was unable to properly consider our warning as a result of her intellectual limits. In any case, her decision that we had to respect, was a great challenge for us. The course of pregnancy was complicated by the underlying disease in the form of recurrent lung infection, chronic respiratory insufficiency, as well as by the patient's mental condition. In addition, she got pregnant shortly after acute respiratory failure requiring artificial pulmonary ventilation and admission to ICU of the Department of Anesthesiology and Intensive Care. The fetus was developing well until the 25 g.w.; the combined I. trimester screening and ultrasound screening in the 20 g.w. were negative and did not reveal any pathology in the fetus. After the 22 g.w. we observed progressive polyhydramnios, which is repeatedly described in pregnant myopathy patients in the literature [9]. After the 25 g.w. we observed the intrauterine stagnation of fetal growth. We had to decide until what stage it would be safe for the mother to maintain her pregnancy. Based on evaluation of the course of the pregnancy we decided to schedule its termination for 30 g.w., taking advantage of a relatively satisfactory clinical and mental stabilization of the patient. It has to be pointed out that without an excellent standard of neonatal care in the Perinatology Center in Ústí nad Labem, similarly as in the whole Czech Republic, such decision would be impossible or, at least, highly risky. In the end, we were even forced by circumstances to terminate the pregnancy a week earlier than planned. Special thanks should also be extended to the whole multidisciplinary team who took care of the patient and her baby [2, 4, 5, 8, 9]. This is a case report of the first pregnancy of a woman with a congenital type of nemaline myopathy in the Czech Republic The case was further complicated by the ongoing Covid 19 pandemic. The treatment outcome of both the mother and the baby seems to be highly promising.

\section{References}

[1] Vinotha, T, Ruby, J.: Nemaline myopathy and pregnancy: A challenge indeed. Neurology India, 2012, 60 (5); 524-525.

[2] Kanani, N.: Pregnancy in a Patient with Nemaline Myopathy. Obstetric Anesthesia Digest. December 2007, 27 (4); 218. 
[3] Berkenstadt, M., Pode-Shakked, B. et al.: LMOD3-Associated Nemaline Myopathy Prenatal Ultrasonographic, Pathologic, and Molecular Findings. J Ultrasound Med 2018; 37: 18271833 .

[4] Radke J, Stenzel W, Goebel HH. Radke J, et al. Corrigendum to "Recently Identified Congenital Myopathies" Semin Pediatr Neurol. 2019 Dec; 32.

[5] Rudnik-Schöneborn, S., Wallgren-Pettersson, C.: Pregnancy and Delivery in Women with Congenital Myopathies Semin Pediatr Neurol 2019 Apr, 29; 23-29.

[6] Eskandar, O., Eckford, S.: Pregnancy in a Patient With Nemaline Myopathy. Obstet Gynecol 2007 Feb; 109 (2 Pt2): $501-4$.

[7] Kuwata T, Matsubara S, Ohkusa T, Yada Y, Suzuki M. Kuwata T, et al. Decreased Fetal Movement Prompts Investigation of prenatal/neonatal Nemaline Myopathy: The Possible Merit of
Fetal Movement CountJ Obstet Gynaecol Res. 2011 Jul; 37 (7): 921-5.

[8] Rudnik-Schöneborn S, Wallgren-Pettersson C. Pregnancy and Delivery in Women With Congenital Myopathies. Semin Pediatr Neurol. 2019 Apr; 29: 23-29.

[9] Berkenstadt M, Pode-Shakked B, Barel O, Barash H, Achiron R, Gilboa Y, Kidron D, Raas-Rothschild A. J LMOD3Associated Nemaline Myopathy: Prenatal Ultrasonographic, Pathologic, and Molecular Findings. Ultrasound Med. 2018 Jul; 37 (7): 1827-1833.

[10] Yeung KS, Yu FNY, Fung CW, Wong S, Lee HHC, Fung STH, Fung GPG, Leung KY, Chung WH, Lee YT, Ng VKS, Yu MHC, Fung JLF, Tsang MHY, Chan KYK, Chan SHS, Kan ASY, Chung BHY. The KLHL40 c.1516A $>$ C is a Chinesespecific founder mutation causing nemaline myopathy 8: Report of six patients with pre- and postnatal phenotypes. Mol Genet Genomic Med. 2020 Jul; 8 (7): e1229. 